\title{
Size and crack length effects on fracture toughness of polycrystalline graphite
}

\author{
J. Akbardoost ${ }^{*}$
}

Faculty of Engineering, Kharazmi University, Mofatteh Avenue, P.O. Box 15719-14911, Tehran, Iran

\begin{tabular}{l}
\hline A R T I C L E I N F O \\
\hline Article history: \\
Received January 20, 2014 \\
Received in Revised form \\
April, 10, 2014 \\
Accepted 25 April 2014 \\
Available online \\
24 April 2014 \\
\hline Keywords: \\
Polycrystalline graphite \\
Fracture toughness \\
Size effect \\
Crack length effect \\
Higher order terms \\
Modified maximum tangential \\
stress criterion
\end{tabular}

\section{A B S T R A C T}

In this paper, the effects of specimen size and crack length on the fracture toughness of polycrystalline graphite are studied. The experimental results reported in the previous studies showed that the fracture toughness of graphite increase in bigger specimen. It has been also demonstrated that the fracture toughness of graphite is nearly identical in specimens with crack length ratio less than 0.7 but decreases for grater crack length ratios. To justify the size and crack length dependency of fracture toughness, the modified form of maximum tangential stress (MMTS) criterion, which makes the use of higher order terms in calculating the stress field around the crack tip is employed. It is shown that the MMTS criterion can provide good estimates for the fracture toughness of graphite obtained from specimen with different sizes. It is also indicated that the MMTS criterion can predict very good the reported experimental fracture toughness data for samples with the crack length ratios less than 0.7 .

\section{Introduction}

Since graphite has good thermal stability, low permeability, high corrosion resistance, good performance in electrical and thermal conductivity and good thermal shock resistance, it is employed frequently in different engineering components such as sliding current connector, refractory graphite parts, sealing rings, carbon brushes, graphite electrodes, heating elements, etc. However, graphite is prone to mechanical or thermal failure, especially when graphite parts contain stress concentrators such as cracks or notches. In other words, the graphite parts might contain cracks, inherent discontinuities and flaws, which play the role of stress raiser and make the graphite parts very susceptible to suddenly fracture. The cracks are often created during the manufacturing or machining processes, or due to the mechanical or thermal loads applied under service conditions. Therefore, it is urgently required to study the mechanical failure of cracked graphite components. Researchers and

* Corresponding author. Tel./Fax: +98 26-4569555

E-mail addresses: Akbardoost@khu.ac.ir (J. Akbardoost) 
engineers have used frequently a fundamental parameter so-called fracture toughness $K_{\text {If }}$ for determining the strength of cracked components. Fracture toughness presents the resistance of materials against crack growth under mode I or opening mode loading. Fracture toughness of graphite is usually measured from standard laboratory specimens with specific geometry, shape and size limits. It is expected that the fracture toughness obtained from laboratory specimen of different sizes and shapes is nearly identical. However, experimental results have shown that the fracture toughness of graphite is significantly dependent on the size, length of initial crack and geometry of specimen (Chi, 2013; Li et al., 2013; Sakai \& Kurita, 1996; Sakai \& Nonoyama, 2005; Yamauchi et al., 2000, 2001; Yoon et al., 2011). For example, Li et al. (2013) showed experimentally that the fracture toughness of NBG graphite obtained from the single-edge-notch beam samples increases by increasing the size of specimen. For another example, Yamauchi et al. (2000, 2001) demonstrated that the fracture toughness of graphite measured from two test configurations including edge-cracked semi-circular bend (SCB) specimen subjected to three-point loading and cracked Brazilian disk (CBD) are significantly different. Moreover, Sakai and Nonoyoma ( 2005) showed that the length of initial crack has the influence on the fracture toughness of graphite when the crack length ratio is more than 0.7. Thus, in order to use the fracture toughness obtained from the laboratory-size specimen for predicting the onset of crack growth in real size and geometry graphite parts, the effects of specimen size, initial crack length and geometry on fracture toughness should be considered.

On the other hand, there are several criteria for investigating the size effects on mode I fracture toughness of quasi-brittle materials such as rocks, concretes, ceramics, etc. For instance, Bazant's size effect law (SEL) proposed by Bazant (1984)is a well-known criterion which has been used frequently for taking into account the specimen size effects on fracture toughness of graphite (Li et al., 2013; Sakai \& Kurita, 1996; Sakai \& Nonoyama 2005). However, almost all of the size effect criteria (e.g. SEL) are not able to consider simultaneously the geometry and crack length effects on the fracture toughness. The aim of this paper is to investigate the size and crack length effects on the fracture toughness of graphite simultaneously. For this purpose, test data reported by Sakai and Kurita(1995) is used. They showed experimentally that the fracture toughness for a type of polycrystalline graphite (IG-11) depends significantly on the size and crack-length of specimen. In order to justify the size and crack-length dependence of fracture toughness, a modified form of the maximum tangential stress (MMTS) criterion is used. This criterion takes into account the influence of the higher order terms in calculating the stress field around the crack tip in addition to the singular terms. As an important parameter in the MMTS criterion, the critical distance $r_{c}$ is assumed to be size dependent and a formula proposed recently by Ayatollahi and Akbardoost ( 2012) is employed for describing the size dependence of $r_{c}$. The values of $K_{I f}$ for specimens with different crack lengths are also predicted using the MMTS criterion based on the constant value of critical distance. It is shown that the MMTS criterion is able to provide good estimates for the fracture toughness of graphite by taking into account the effects of specimen size and the length of initial crack.

\section{Modified MTS criterion}

Based on the classical MTS criterion proposed by Erdogan and Sih (1963), fracture occurs radially from the crack tip and perpendicular to the direction of the maximum tangential stress $\theta_{m}$. Moreover, the crack will be extended when the tangential stress component $\sigma_{\theta \theta}$ along $\theta_{m}$ and at a critical distance $r_{c}$ from the crack tip reaches a critical value $\sigma_{\theta \theta c}$ (Erdogan \& Sih 1963). The tangential stress around the crack tip under pure mode I loading can be written from the William's series expansion (Williams, 1957):

$$
\sigma_{\theta \theta}(r, \theta)=\sum_{n=1}^{\infty} \frac{n}{2} A_{n} r^{\frac{n}{2}-1}\left[\left(\frac{n}{2}+1\right) \cos \left(\frac{n}{2}-1\right) \theta-\left(\frac{n}{2}+(-1)^{n}\right) \cos \left(\frac{n}{2}+1\right) \theta\right]
$$


where $r$ and $\theta$ are the conventional crack tip co-ordinates, $n$ is the order of term in the series expansion and the constant coefficients $A_{n}$ are dependent on the specimen geometry and loading conditions. These coefficients can be generally written in terms of dimensionless parameters $\mathrm{A}_{n}{ }^{*}$ as:

$$
A_{n}=\sigma_{N} w^{(1-n / 2)} A_{n}^{*}
$$

in which $\sigma_{N}$ is the nominal stress and $w$ is a characteristic dimension like the width of single-edge notched beam (SENB) specimens. Moreover, the dimensionless coefficients $A_{n}{ }^{*}$ depend only on the configuration parameters like the crack length ratio $(a / w)$ and the loading span to width ratio $(S / w)$. These parameters are independent of the load and the dimensions of samples. Due to symmetry in mode I loading, the crack growth takes places along the crack direction, i.e. $\theta_{m}=0$. Therefore, the tangential stress $\sigma_{\theta \theta}$ along the fracture direction is obtained from Eq. (1) by setting $\theta=0$ :

$$
\left.\sigma_{\theta \theta}(r, \theta)\right|_{\theta=0}=\frac{A_{1}}{\sqrt{r}}+3 A_{3} \sqrt{r}+5 A_{5} r^{3 / 2}+\ldots
$$

The parameter $\mathrm{A}_{1}$ is related to the mode $\mathrm{I}$ stress intensity factor $K_{I}$ as $A_{1}=K_{I} / \sqrt{2 \pi}$. The conventional MTS criterion (Erdogan \& Sih, 1963) considers only the first stress term and ignores the higher order terms. It has been recently demonstrated that the higher order terms of crack tip asymptotic field are no longer negligible and they should be taken into account to characterize the tangential stress more accurately (Awaji \& Sato, 1978; Ayatollahi \& Akbardoost, 2012; Ayatollahi \& Aliha, 2011; Aliha \& Ayatollahi, 2009, 2013; Mirsayar et al., 2014; Aliha et al., 2010, 2013). By taking the first two terms in Eq. (3) into consideration, the maximum tangential stress component at the critical distance $r_{c}$ can be obtained from:

$$
\sigma_{\theta \theta c}=\frac{A_{1 c}}{\sqrt{r_{c}}}+3 A_{3 c} \sqrt{r_{c}}=\frac{K_{I f}}{\sqrt{2 \pi r_{c}}}+3 A_{3 c} \sqrt{r_{c}}
$$

where $A_{3 c}$ is the critical value of $A_{3}$ and $K_{I f}$ is the critical stress intensity factor or apparent fracture toughness which is considered to be dependent on the size and geometry of cracked specimen. By substituting Eq. (2) into Eq. (4) for $\mathrm{n}=1$ and $\mathrm{n}=3$, Eq. (4) simplifies to:

$$
\sigma_{\theta \theta c}=\frac{K_{I f}}{\sqrt{2 \pi r_{c}}}\left(1+3 \frac{A_{3}^{*}}{A_{1}^{*}} \frac{r_{c}}{w}\right)
$$

According to previous studies and considering the intrinsic features of graphite (Ayatollahi \& Aliha, 2011; Li et al., 2013; Sakai \& Kurita 1996), the critical value of $\sigma_{\theta \theta c}$ can be assumed to be the tensile strength of materials, $f_{t}$. Thus, the mode I brittle fracture occurs when:

$f_{t}=\frac{K_{I f}}{\sqrt{2 \pi r_{c}}}\left(1+3 \frac{A_{3}^{*}}{A_{1}^{*}} \frac{r_{c}}{w}\right)$

In order to use Eq. (6) for predicting the onset of fracture, one should first determine the critical distance $r_{c}$. There are several formulations in the literature for calculating the value of $r_{c}$ (Bazant et al., 1991; Bazant \& Planas 1998; Karihaloo, 1999; Schmidt, 1980). Recently, Ayatollahi and Akbardoost (2012) proposed a modified form of Schmidt's formula (Schmidt, 1980) for determining the value of $r_{c}$. The proposed formula can be written as:

$$
r_{c}=\left[\frac{f_{t} \sqrt{2 \pi} \pm \sqrt{2 \pi f_{t}^{2}-12 \frac{A_{3}^{*}}{A_{1}^{*}} \frac{K_{\text {If }}{ }^{2}}{w}}}{6 \frac{A_{3}^{*}}{A_{1}^{*} \frac{K_{I f}}{w}}}\right]^{2}
$$


In this formula, $K_{I f}$ is the mode I fracture resistance, $A_{1}{ }^{*}$ and $A_{3}{ }^{*}$ are the dimensionless parameters for the coefficients of first and third stress terms in pure mode I loading. It has been also shown (Bazant \& Planas, 1998; Karihaloo, 1995) that the value of $r_{c}$ depends on the size of specimen and increases by increasing the specimen size. Therefore, the size dependent value of $r_{c}$ should be considered in the proposed method. Here, a simplified formula proposed recently by Ayatollahi and Akbardoost (2012) is used for describing the size dependency of $r_{c}$ :

$$
r_{c}=\frac{A}{1+\frac{B}{w}}
$$

where the constant coefficients $A$ and $B$ are calculated by a linear regression on fracture resistance obtained from mode I tests conducted on specimens of different sizes. For calculating $A$ and $B$, the value of $r_{c}$ corresponding to each specimen size is first determined by replacing the fracture toughness $\left(K_{I f}\right)$ obtained from the experiment into Eq. (7). Then a linear regression based on $Y=M X$ $+Q$, in which:

$$
Y=\frac{1}{r_{c}}, M=\frac{B}{A}, X=\frac{1}{w}, Q=\frac{1}{A}
$$

is used (see more details in Ayatollahi and Akbardoost (2012)). By simplifying Eq. (6), the variations of fracture toughness $K_{I f}$ versus the specimen size can be obtained from:

$$
K_{I f}=\frac{f_{t} \sqrt{2 \pi r_{c}}}{\left(1+3 \frac{A_{3}^{*}}{A_{1}^{*} \frac{r_{c}}{w}}\right)}
$$

in which the value of $r_{c}$ is determined according to specimen size from Eq. (8). Since the proposed criterion is a modified form of the MTS criterion, it is named the MMTS criterion. In the next section, the size dependent values of fracture toughness for a polycrystalline graphite reported by Sakai and Kurita (1995) will be justified using the MMTS criterion. It will be also shown that the fracture toughness of graphite obtained from specimens of different crack lengths can be predicted using the MMTS criterion.

\section{Graphite experiments}

Sakai and Kurita (1995)conducted several experiments on the single-edge notched beam (SENB) for investigating the dependency of size and crack length on the fracture toughness of graphite. The schematic of the SENB sample and geometric notations are shown in Fig. 1. The graphite employed by Sakai and Kurita (1995) was an isotropic polycrystalline graphite (IG- 11) with the following mechanical properties: bulk density of $1.76 \mathrm{~g} / \mathrm{cm}^{3}$,Young's modulus of $9 \mathrm{GPa}$ and tensile strength of $27 \mathrm{MPa}$. The samples were classified into two categories: 1) the specimen having similar geometry but different sizes, 2) the specimens with similar characteristic dimension, i.e. $w$, but different crack lengths $a$. In the first category, the widths of specimens were $w=2.5,5,7.5,10,12.5,17.5,20 \mathrm{~mm}$ and their crack length ratio was constant and equal to 0.5 . The second category was performed on the specimens with the constant width of $12.5 \mathrm{~mm}$ but different crack lengths of $a=1.25,2.5,3.75,5$, $6.25,7.5,8.75,10,11.25 \mathrm{~mm}$. For all specimens, the thickness $t$ was $10 \mathrm{~mm}$ and the span to width ratio $S / w$ was equal to 4 .The dimensions of samples and loading conditions for the first and second test categories are listed in Table 1 and Table 2, respectively.

The fracture toughness for the SENB samples is often calculated from:

$$
K_{I f}=\frac{3 P_{u} S}{2 w^{2} t} \sqrt{2 \pi w} A_{1}^{*},
$$


where $P_{u}$ is fracture load. Sakai and Kurita (1995) did not report the fracture loads of tested specimens and they only presented the values of fracture toughness as displayed in Table 1 and Table 2. As seen from Table 1, the fracture toughness of graphite depends on the specimen size and increases by increasing the size of specimen. Meanwhile, the fracture toughness of tested graphite is nearly constant for specimens larger than $w=12.5 \mathrm{~mm}$. Therefore, one can obtained the sizeindependent value of $K_{\text {If }}$ by testing the specimen larger than the width of $12.5 \mathrm{~mm}$. Table 2 also shows that the fracture toughness of graphite is nearly constant for specimens with crack lengths of $a=1.25,2.5,3.75,5,6.25$ and $8.75 \mathrm{~mm}$ and decreases for larger crack lengths. It means that the fracture toughness of graphite depends on the crack length when the crack length ratio is larger than 0.7 .

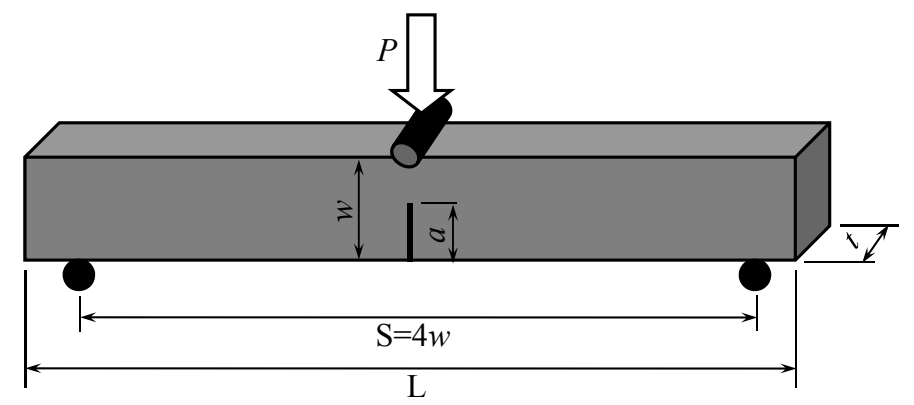

Fig. 1. The schematic of SENB specimen and its dimension notations

Table 1. Graphite specimens and their fracture parameters for size effect analysis

\begin{tabular}{ccc}
$\begin{array}{c}\text { Category I (Size effect analysis) } \\
\text { Specimen Dimensions (in mm) }\end{array}$ & $\begin{array}{c}S / w=0.4, a / w=0.5 \\
K_{I f}\left(\mathrm{MPa} . \mathrm{m}^{0.5}\right) \\
(\text { Sakai and Kurita 1995) }\end{array}$ & $\begin{array}{c}r_{c} \\
(\mathrm{~mm})\end{array}$ \\
\hline $20 \times 2.5 \times 10$ & 0.59 & 0.062 \\
$30 \times 5 \times 10$ & 0.62 & 0.075 \\
$40 \times 7.5 \times 10$ & 0.65 & 0.085 \\
$50 \times 10 \times 10$ & 0.68 & 0.094 \\
$60 \times 12.5 \times 10$ & 0.7 & 0.102 \\
$80 \times 17.5 \times 10$ & 0.705 & 0.104 \\
$90 \times 20 \times 10$ & 0.71 & 0.106 \\
\hline
\end{tabular}

Table 2. Graphite specimens and their fracture parameters for crack length effect analysis Category II (crack length effect analysis) $S / w=0.4, w=12.5 \mathrm{~mm}$

Specimen Dimensions (in mm)

$(L \times a \times t)($ Sakai and Kurita 1995)

$60 \times 1.25 \times 10$

$60 \times 2.5 \times 10$

$60 \times 3.75 \times 10$

$60 \times 5 \times 10$

$60 \times 6.25 \times 10$

$60 \times 7.5 \times 10$

$60 \times 8.75 \times 10$

$60 \times 10 \times 10$

$60 \times 11.25 \times 10$

$$
K_{\text {If }}\left({\left.\mathrm{MPa} . \mathrm{m}^{0.5}\right)}^{0}\right.
$$

(Sakai and Kurita 1995)

0.7

0.68

0.73

0.72

0.7

0.69

0.69

0.65

0.63 


\section{Results and discussion}

In order to employ the MMTS criterion for size and crack length effects studies, the dimensionless form of the coefficients of first two terms in crack tip stress field, i.e. $A_{1}{ }^{*}$ and $A_{3}{ }^{*}$, should be known. The non-dimensional parameters $A_{1}{ }^{*}$ and $A_{3}{ }^{*}$, which are functions of $S / w$ and $a / w$, can be determined analytically for simple geometries and numerically for complicated cracked bodies. Karihaloo and Xiao (2001) proposed the following equations for calculating the values of $A_{1}{ }^{*}$ and $A_{3}{ }^{*}$ for three-point bend specimens with $S / w$ of 4 :

$$
\begin{aligned}
& A_{1}^{*}(\alpha)=\frac{\sqrt{\alpha}\left(1.9+0.41 \alpha+0.51 \alpha^{2}-0.17 \alpha^{3}\right)}{\sqrt{2 \pi}(1-\alpha)^{3 / 2}(1+3 \alpha)} \\
& A_{3}^{*}(\alpha)=0.6534-9.2406 \alpha+49.515 \alpha^{2}-153.97 \alpha^{3}+233.48 \alpha^{4}-148.73 \alpha^{5}
\end{aligned}
$$

where $\alpha$ in Eq. (11) and Eq. (12) is crack length ratio, i.e. $\alpha=a / w$. These equations will be used for predicting the fracture onset of graphite specimens by taking into account the effects of specimen size and crack length.

\subsection{Size effect analysis}

After calculating the values of $A_{1}{ }^{*}$ and $A_{3}{ }^{*}$ in Eq.(9), the value of critical distance should be determined. The values of $r_{c}$ for each tested specimens are obtained from Eq. (7). For using this formula, the tensile strength $\left(f_{t}\right)$ and mode I fracture toughness $\left(K_{I f}\right)$ should be known. The tensile strength of IG-11 graphite $f_{t}$ was found to be 27MPafrom(Sakai and Kurita 1995). The mode I fracture toughness $\left(K_{I f}\right)$ was also extracted from Table 1 related to the size of specimen. The values of $r_{c}$ calculated from Eq. (7) for each tested samples are shown in Table 1. According to this Table, the values of $r_{c}$ depends on the specimen size and increases for bigger samples. Therefore, the size dependency of $r_{c}$ should be considered. Eq. (8) is employed here for describing the variations of $r_{c}$ versus the specimen size. For using Eq. (8), the values of $A$ and $B$ should be determined from a linear regression of the test results. The linear regression plot, as shown in Fig. 2, gives $A=0.106 \mathrm{~mm}$ and $B$ $=1.806 \mathrm{~mm}$. It is noteworthy that the values of $A$ and $B$ are calculated based on the first four test data and the experimental results for other specimen sizes (i.e. $w=12.5,17.5$ and $20 \mathrm{~mm}$ ) are assumed to be unknown and will be predicted using the MMTS criterion. After the calculation of $A$ and $B$, the critical distance can be written as a function of specimen size. Fig. 3 illustrates the variations of $r_{c}$ versus the specimen size obtained from Eq. (8).

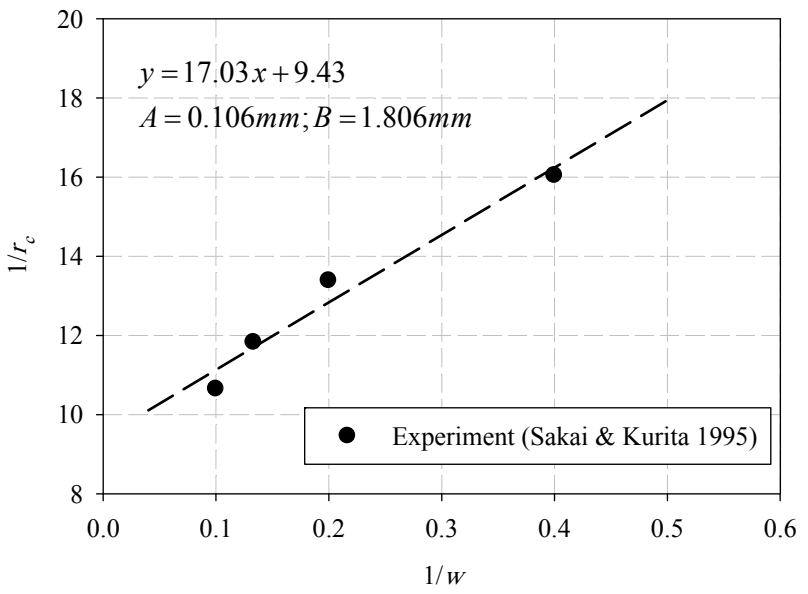

Fig. 2. Linear regression for calculating $A$ and $B$ in Eq. (8)



Fig. 3. The variations of critical distance versus the specimen size for graphite 
Now, the size-dependent behavior of $K_{\text {If }}$ can be presented using Eq. (9)as an asymptotic curve. Fig. 4 displays the variations of $K_{\text {If }}$ relative to the specimen size together with the experimental results. As shown in this Figure, the MMTS criterion can provide good estimates for the fracture toughness of specimens whit width of $w=12.5,17.5$ and $20 \mathrm{~mm}$ which were assumed to be unknown. Moreover, Fig. 4 indicates that the material fracture resistance $K_{\text {If }}$ for infinitely large specimens is independent of specimen size and approaches a nearly constant value. The size-independent value of $K_{I f}$ can be obtained directly from Eq. (9) by taking was infinity. According to Eq. (9), when $w \rightarrow \infty$ the value of $K_{I f}$ is calculated as $0.7 \mathrm{MPa}^{0.5}$. This value of $K_{I f}$, which is denoted by $K_{I c}$ can be considered as the inherent fracture toughness of IG-11 graphite. One might argue that the value of $K_{I c}$ for IG-11 is less than those values obtained from the specimen sizes of $w=17.5$ and $20 \mathrm{~mm}$ (see Table 1). This is because $K_{I c}$ was calculated based on the linear regression of 4test data and the more accurate value of $K_{I c}$ can be achieved by using all test results. If all test data were used in the linear regression for determining the values of $A$ and $B$, the value of $K_{I c}$ would be calculated as $K_{I c}=0.723$ MPa.m.

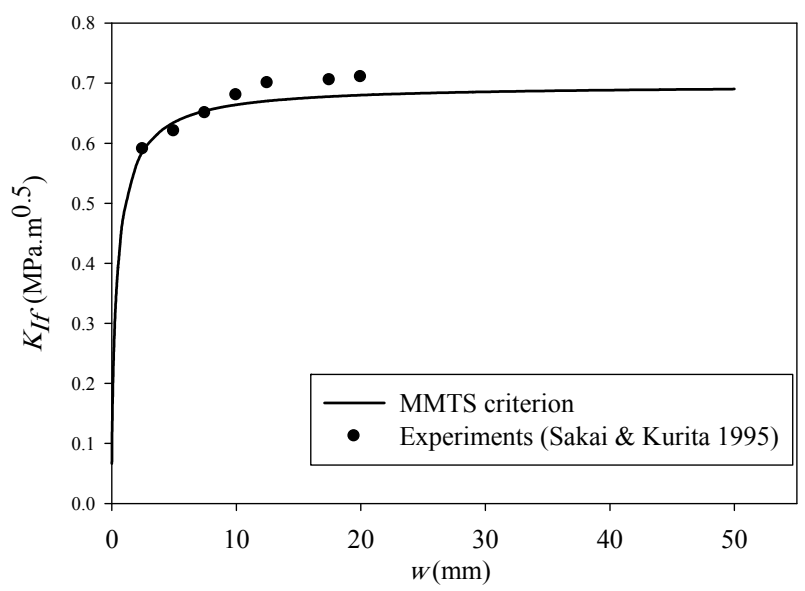

Fig. 4. The variations of fracture toughness of graphite with respect to the specimen size

\subsection{Crack length effect analysis}

The MMTS criterion or Eq. (6)is employed here to predict the fracture toughness of graphite obtained from the second test category. The specimens in the second test category have the same width but different crack lengths (see Table 2). By considering the tensile strength $f_{t}$ as a constant material property, the left hand side of Eq. (6) would be a constant value for any given material. Thus, the following relation can be written for two specimens with different crack length ratios:

$$
f_{t}=\left.\frac{K_{I f}}{\sqrt{2 \pi r_{c}}}\left(1+3 \frac{A_{3}^{*}}{A_{1}^{*}} \frac{r_{c}}{w}\right)\right|_{(a / w)_{1}}=\frac{K_{I f}}{\sqrt{2 \pi r_{c}}}\left(1+\left.3 \frac{A_{3}^{*}}{A_{1}^{*}} \frac{r_{c}}{w}\right|_{(a / w)_{2}}\right.
$$

In order to derive a relation between the values of mode I fracture toughness for two specimens with different crack lengths, Eq. (13) can be rewritten as: 


$$
\frac{\left.K_{I f}\right|_{(a / w)_{1}}}{\left.K_{I f}\right|_{(a / w)_{2}}}=\frac{\left.\frac{1}{\sqrt{2 \pi r_{c}}}\left(1+3 \frac{A_{3}^{*}}{A_{1}^{*}} \frac{r_{c}}{w}\right)\right|_{(a / w)_{2}}}{\left.\frac{1}{\sqrt{2 \pi r_{c}}}\left(1+3 \frac{A_{3}^{*}}{A_{1}^{*}} \frac{r_{c}}{w}\right)\right|_{(a / w)_{1}}}
$$

The dimensionless parameters $A_{1}{ }^{*}$ and $A_{3}{ }^{*}$ in Eq. (14) can be respectively obtained from Eq. (11) and Eq. (12) for the SENB specimens with different crack length ratio $\alpha$. Table 3 shows these parameters for each specimen in the second test category. Since all specimens in this test category have the same width of $12.5 \mathrm{~mm}$, the critical distance $r_{c}$ in Eq. (14) is taken to be as $0.102 \mathrm{~mm}$ according to the value obtained from SENB specimen of $w=12.5 \mathrm{~mm}$. By replacing the values of $A_{3}{ }^{*} / A_{1}{ }^{*}$ corresponding to the specimens of the second test category and the value of $r_{c}=0.102 \mathrm{~mm}$ in Eq. (14), the fracture toughness $K_{I f}$ for these specimens are estimated as shown in Table 3. It should be noted that the reference fracture toughness in Eq. (14) is taken to be $0.7 \mathrm{MPa} . \mathrm{m}^{0.5}$ obtained from specimen with the width of $w=12.5 \mathrm{~mm}$.

Table 3. The values of dimensionless parameters and fracture toughness predicted by MMTS criterion for graphite specimens in the second test category

\begin{tabular}{|c|c|c|c|c|c|c|c|}
\hline \multirow{2}{*}{$\begin{array}{l}\text { Specimen Dimensions } \\
(L \times a \times t)(\text { in } \mathrm{mm})\end{array}$} & \multirow{2}{*}{$\alpha=a / w$} & \multirow{2}{*}{$A_{1}{ }^{*}$} & \multirow{2}{*}{$A_{3}{ }^{*}$} & \multirow{2}{*}{$A_{3}{ }^{*} / A_{1}{ }^{*}$} & \multicolumn{3}{|c|}{$\begin{array}{c}K_{I f} \\
\left(\mathrm{MPa} . \mathrm{m}^{0.5}\right)\end{array}$} \\
\hline & & & & & $\begin{array}{c}\text { Experiment } \\
\text { (Sakai and Kurita 1995) }\end{array}$ & MMTS & $\begin{array}{c}\text { Error } \\
(\%)\end{array}$ \\
\hline $60 \times 1.25 \times 10$ & 0.1 & 0.221 & 0.092 & 0.418 & 0.7 & 0.672 & 4 \\
\hline $60 \times 2.5 \times 10$ & 0.2 & 0.312 & -0.120 & -0.385 & 0.68 & 0.685 & -1 \\
\hline $60 \times 3.75 \times 10$ & 0.3 & 0.405 & -0.290 & -0.715 & 0.73 & 0.690 & 5 \\
\hline $60 \times 5 \times 10$ & 0.4 & 0.527 & -0.520 & -0.988 & 0.72 & 0.695 & 3 \\
\hline $60 \times 6.25 \times 10$ & 0.5 & 0.706 & -0.890 & -1.261 & 0.7 & 0.700 & 0 \\
\hline $60 \times 7.5 \times 10$ & 0.6 & 1.000 & -1.629 & -1.629 & 0.69 & 0.707 & -2 \\
\hline $60 \times 8.75 \times 10$ & 0.7 & 1.559 & -3.303 & -2.119 & 0.69 & 0.716 & -4 \\
\hline $60 \times 10 \times 10$ & 0.8 & 2.895 & -6.985 & -2.413 & 0.65 & 0.721 & -11 \\
\hline $60 \times 11.25 \times 10$ & 0.9 & 8.275 & -14.437 & -1.745 & 0.63 & 0.709 & -12 \\
\hline
\end{tabular}

Table 3 indicates that the MMTS criterion can provide good estimates for the fracture toughness obtained from specimens with the crack length ratios of $\alpha=0.1$ to 0.7. It is also shown in Table 3 that there is a discrepancy of more than $10 \%$ between the values of fracture toughness obtained experimentally from specimens with crack length ratios of 0.8 and 0.9 and those values predicted by the MMTS criterion. The main reason for this discrepancy can be that the critical distance employed in this section is taken to be constant for different crack length ratios. The previous studies (Bazant \& Planas 1998; Wittmann \& Hu 1991) have shown that the fracture process zone around the crack tip is affected by the back boundary when $\alpha>0.7$. Therefore, the MMTS criterion cannot predict the accurate fracture toughness for specimens with $\alpha>0.7$.

It is noted that the stress field around the crack tip is affected by the back boundaries when $\alpha$ is larger than 0.7 and thus the William's series expansions cannot characterize the near tip stress components. Therefore, the laboratory specimens with $\alpha>0.7$ are not usually employed for determining the fracture properties of materials(Hu \& Wittmann, 2000). Moreover, the real graphite structures will be failed before the crack length ratios reaches to 0.7 and, therefore, the components with $\alpha>0.7$ are practically invalid. Consequently, one can concluded that the MMTS criterion is able to predict the onset of fracture in real graphite structures.

\section{Conclusions}

The size and crack length effects on the fracture toughness of graphite was studied using the modified maximum tangential stress (MMTS) criterion which takes into account the $A_{3}$ term in the 
Williams series expansion in addition to the conventional singular term of stress. The critical distance $r_{c}$ is also assumed to be size dependent in the MMTS criterion when the size effect on fracture toughness is investigated. It was shown that the theoretical estimates obtained from the MMTS criterion are in good agreement with the experimental results reported in the previous studies. To analyze the crack length effect on the mode I fracture resistance of graphite by using MMTS, the critical distance $r_{c}$ was taken to be the same value for specimens with different crack lengths. Comparison between the fracture toughness predicted by MMTS and those experimental values reported in previous studies indicated that the MMTS criterion can provide good estimates for fracture toughness of specimen with crack length ratios less than 0.7 , i.e. $\alpha \leq 0.7$. Since the crack tip is close to the back boundaries for $\alpha>0.7$ (which affects the stress field around the crack tip), the MMTS criterion is not able to predict accurately the value of $K_{I f}$ obtained from the experimental results.

\section{References}

Aliha, M. R. M., \& Ayatollahi, M. R. (2009). Brittle fracture evaluation of a fine grain cement mortar in combined tensile-shear deformation. Fatigue \& Fracture of Engineering Materials \& Structures, 32(12), 987-994.

Aliha, M. R. M., \& Ayatollahi, M. R. (2013). Two-parameter fracture analysis of SCB rock specimen under mixed mode loading. Engineering Fracture Mechanics, 103, 115-123.

Aliha, M. R. M., Hosseinpour, G. R., \& Ayatollahi, M. R. (2013). Application of Cracked Triangular Specimen Subjected to Three-Point Bending for Investigating Fracture Behavior of Rock Materials. Rock Mechanics and Rock Engineering, 46(5), 1023-1034.

Aliha, M. R. M., Ayatollahi, M. R., Smith, D. J., \& Pavier, M. J. (2010). Geometry and size effects on fracture trajectory in a limestone rock under mixed mode loading. Engineering Fracture Mechanics, 77(11), 2200-2212.

Awaji, H., \& Sato, S. (1978). Combined mode fracture toughness measurement by the disk test. Journal of Engineering Materials and Technology, 100(2), 175-182.

Ayatollahi, M. R., \& Akbardoost, J. (2012). Size effects on fracture toughness of quasi-brittle materials-A new approach. Engineering Fracture Mechanics, 92, 89-100.

Ayatollahi, M. R., \& Aliha, M. R. M. (2011). Fracture analysis of some ceramics under mixed mode loading. Journal of the American Ceramic Society, 94(2), 561-569.

Bazant, Z. P. (1984). Size effect in blunt fracture: concrete, rock, metal. Journal of Engineering Mechanics, 110(4), 518-535.

Bažant, Z. P., Gettu, R., \& Kazemi, M. T. (1991, January). Identification of nonlinear fracture properties from size effect tests and structural analysis based on geometry-dependent R-curves. In International Journal of Rock Mechanics and Mining Sciences \& Geomechanics Abstracts (Vol. 28, No. 1, pp. 43-51). Pergamon.

Bazant, Z. P., \& Planas, J. (1997). Fracture and size effect in concrete and other quasibrittle materials (Vol. 16). CRC press.

Chi, S. H. (2013). Specimen size effects on the compressive strength and Weibull modulus of nuclear graphite of different coke particle size: IG-110 and NBG-18. Journal of Nuclear Materials, 436(1), 185-190.

Erdogan, F., \& Sih, G. C. (1963). On the crack extension in plates under plane loading and transverse shear. Journal of basic engineering, 85(4), 519-525.

$\mathrm{Hu}, \mathrm{X}$. , \& Wittmann, F. (2000). Size effect on toughness induced by crack close to free surface. Engineering fracture mechanics, 65(2), 209-221.

Karihaloo, B. L. (1995). Tension softening diagrams and longitudinally reinforced beams. Fracture of brittle disordered materials: concrete, rock and ceramic, 35-50.

Karihaloo, B. L. (1999). Size effect in shallow and deep notched quasi-brittle structures. International Journal of Fracture, 95(1-4), 379-390. 
Karihaloo, B. L., \& Xiao, Q. Z. (2001). Higher order terms of the crack tip asymptotic field for a notched three-point bend beam. International Journal of Fracture, 112(2), 111-128.

Li, H., Li, J., Singh, G., \& Fok, A. (2013). Fracture behavior of nuclear graphite NBG-18. Carbon, 60, 46-56.

Mirsayar, M. M., Aliha, M. R. M., \& Samaei, A. T. (2014). On fracture initiation angle near bimaterial notches-Effects of first non-singular stress term. Engineering Fracture Mechanics, 119, 124-131.

Sakai, M. \& Kurita, H. (1995) Deformation and fracture in the frontal process zone and the crackface contact region of a polycrystalline graphite. In: Baker G, Karihaloo BL (eds) Fracture of Brittle Disordered Materials: Concrete, Rock and Ceramics. E \& FN Spon, London pp 227-245

Sakai, M., \& Kurita, H. (1996). Size-Effect on the Fracture Toughness and the R-Curve of Carbon Materials. Journal of the American Ceramic Society, 79(12), 3177-3184.

Sakai, M., \& Nonoyama, R. (2005). Nonlinear Fracture of a Polycrystalline Graphite-Size-Effect Law and Irwin's Similarity. In Fracture Mechanics of Ceramics (pp. 337-351). Springer US.

Schmidt, R. A. (1980, January). A microcrack model and its significance to hydraulic fracturing and fracture toughness testing. In The 21st US Symposium on Rock Mechanics (USRMS). American Rock Mechanics Association.

Williams, M. L. (1957). On the stress distribution at the base of a stationary crack. Journal of Applied Mechanics, 24, 109-114.

Wittmann, F. H. \& Hu, X. (1991). Fracture process zone in cementitious materials. International Journal of Fracture, 51(1), 3-18.

Yamauchi, Y., Nakano, M., Kishida, K. \& Okabe, T. (2000). Measurement of fracture toughness for brittle materials under mixed mode impact loading using center-notched disk specimen. Journal of the Society of Materials Science, Japan, 49 (12), 1324-1329.

Yamauchi, Y., Nakano, M., Kishida, K. \& Okabe, T. (2001). Measurement of mixed-mode fracture toughness for brittle materials using edge-notched half-disk specimen. Journal of the Society of Materials Science, Japan, 50 (3), 224-229.

Yoon, J. H., Byun, T. S., Strizak, J. P. \& Snead, L. L. (2011). Characterization of tensile strength and fracture toughness of nuclear graphite NBG-18 using subsize specimens. Journal of Nuclear Materials, 412 (3), 315-320. 